\title{
Amino acid profiling as a method of discovering biomarkers for diagnosis of neurodegenerative diseases
}

\author{
Edyta Socha ${ }^{1} \cdot$ Marcin Koba ${ }^{1} \cdot$ Piotr Kośliński ${ }^{1}$
}

Received: 11 October 2018 / Accepted: 22 January 2019 / Published online: 6 February 2019

(c) The Author(s) 2019

\begin{abstract}
The trend of growing population of $60+$ years old people is visible in most of the highly developed European countries. Recently researchers have shown an increased interest in aging-associated diseases including neurological disorders. Neurodegenerative diseases are a very important clinical problem for several reasons. One of the key aspects are: frequency of occurrence as well as difficulties in the diagnosis, therapeutic problems and care of elderly patients. Furthermore, the very important point is the significant decrease of quality of life of untreated patients and late-stage diagnosis of the disease. It is crucial to develop a new, faster, high specificity and more sensitive diagnostic technology. Metabolomic profiling is a new, promising field of systems biology which may be applied in screening, diagnosis, disease typing and monitoring of treatment. It is a biochemical approach for biomarker discovery. Amino acids (AA) play very integral roles in the central nervous system as neurotransmitters, regulators of metabolism and neuromodulators. Research presented in this publication is focused on patients with Parkinson's disease, Alzheimer disease, and elderly patients. In all analyzed cases significant changes in the amino acid profile in patients comparing to healthy controls were observed. This study indicates potential of amino acid profiling as a method for diagnosis.
\end{abstract}

Keywords Metabolomics $\cdot$ Amino acid profiling $\cdot$ Neurodegenerative diseases $\cdot$ Cognitive impairment $\cdot$ Biomarker

\section{Introduction}

Intensification of aging populations is an unquestionable success of modern medicine on one hand, and a huge challenge and risk on the other. Significant changes in the demographic structure of most European countries are resulting in more and more consequences. The trend of growing population of $60+$ years old people is visible in most of the highly developed European countries. Recently, researchers have shown an increased interest in aging-associated diseases. World Health Organization discusses and describes the increasing global public health importance of common neurological disorders such as dementia, multiple sclerosis, neuroinfections, pain associated with neurological disorders,

Handling Editor: J. D. Wade.

Marcin Koba

kobamar@cm.umk.pl

1 Department of Toxicology, Faculty of Pharmacy, Collegium Medicum of Nicolaus Copernicus University, Bydgoszcz, Poland
Alzheimer Disease, Parkinson's disease, neurological disorders associated with malnutrition stroke and traumatic brain injuries. Age is the main risk factor for numerous diseases whose importance increases with the aging of society. Neurodegenerative diseases are characterized by the slow loss of neurons in the discrete areas of the central nervous system, which can give rise to disorders in specific brain functions. Consequences of neurodegenerative diseases are deficits in memory, movement, cognition et cetera. This group of chronic, progressive diseases includes, e.g., Alzheimer's disease (AD), dementia, Parkinson's disease (PD), multiple sclerosis, amyotrophic lateral sclerosis (ALS), multiple system atrophy and Huntington's disease. Most of neurodegenerative diseases are considered to be slowly progressive brain disorders that begin long before the onset of manifestations (Hui-Ming and Jau-Shyong 2008; Hoffman et al. 2017). Neurodegenerative disorders including dementia syndrome are very important clinical problems for several reasons. One of the key aspects is frequency of occurrence (epidemiology) as well as difficulties in the diagnosis, therapeutic problems and care of elderly patients. Furthermore, the very important point is the significant decrease of quality 
of life of untreated patients and late-stage diagnosis of the disease. It is crucial to develop a new, faster, high specificity and more sensitive diagnostic technologies (Tables 1, 2, 3).

Metabolomic profiling is a new, promising field of systems biology which may apply in screening, diagnosis, disease typing and monitoring of treatment. It is a biochemical approach for biomarker discovery. Amino acids (AA) play very integral roles in the central nervous system as a neurotransmitters, regulators of metabolism and neuromodulators. AAs are important in both mental and physical functions as regulators and nutrients (Figura et al. 2018; Adachi et al. 2018). Recently, researchers indicate the amino acid profile as a potential marker for detection of numerous groups of diseases (Roth et al. 2011; Figura et al. 2018; Corso et al. 2017). One of the most significant current challenges of the research is to identify highly specific, sensitive, reliable, easy-to-measure biomarkers that are able to detect disease in its early stage (Hasim et al. 2013; Roth et al. 2011). Metabolomics methods using amino acid detection have many potential applications. These applications include understanding the pathogenesis, gene modification, and detecting diseases. These methods apply high-technology techniques such as highperformance liquid chromatography (HPLC)/mass spectrometry (MS) and nuclear magnetic resonance (NMR) to measure metabolites in biological material such as body fluids, cells, tissues and organs (Corso et al. 2017; Inoue et al. 2013; Zhao et al. 2017).

This review is concentrated on analyzing studies of the most common neurodegenerative diseases. Inventing effective methods of early diagnosis is particularly crucial in case of diseases with such characteristics.

Table 1 Results of three studies on amino acid profiles in patients with Alzheimer's disease comparing to healthy controls (Nai-jing et al. 2010; Corso et al. 2017; Fonteh et al. 2007)

\begin{tabular}{|c|c|c|c|}
\hline Authors & Year & Results & Comments \\
\hline Nai-jing et al. & 2010 & $\begin{array}{l}\text { Significant decrease in concentration of tryptophan in } \\
\text { patients with } \mathrm{AD} \text { comparing to healthy control }(\mathrm{HC})\end{array}$ & $\begin{array}{l}\text { AD group }-N=20 \\
\mathrm{HC} \text { group }-N=20 \\
\text { Free of dementia volunteers in healthy group did not have } \\
\text { any neurological and cognitive disease }\end{array}$ \\
\hline Corso et al. & 2017 & $\begin{array}{l}\text { Progressive decrease in concentrations of glutamate, aspar- } \\
\text { tate and phenylalanine } \\
\text { Progressive increase in concentrations of citrulline and } \\
\text { homocitrulline }\end{array}$ & $\begin{array}{l}\text { Investigated material from } 4 \text { groups of patients: } \\
\text { Participants with probable AD-N=29 } \\
\text { Patients with mild cognitive impairment (MCI) }-N=18 \\
\text { Patients with subjective memory complaint (SMC) }-N=24 \\
\text { Healthy subjects }-N=46\end{array}$ \\
\hline Fonteh et al. & 2007 & $\begin{array}{l}\text { Significant increase in concentration of urine histidine, } \\
\text { 3-methyl-histidine and carnosine (dipeptide molecule, } \\
\text { made up of the amino acids beta-alanine and histidine) in } \\
\text { patients with AD than in control group } \\
\text { Significant decrease in concentration of histidine in } \\
\text { cerebrospinal fluid from AD patients than that of control } \\
\text { group }\end{array}$ & $\begin{array}{l}\text { AD group }-N=8 \\
\text { HC group- }-N=8\end{array}$ \\
\hline
\end{tabular}

Table 2 Results of study on amino acid profiles in patients with advanced Parkinson's disease comparing to patients with early Parkinson's disease (Figura et al. 2018)

\begin{tabular}{llll}
\hline Authors & Year & Results & Comments \\
\hline Figura et al. & 2018 & Significant decrease in concentration of alanine, arginine, threonine and phenylalanine & $\begin{array}{l}\text { Patients in early PD— } N=22 \\
\text { Patients in advanced PD— }-N=51\end{array}$ \\
\hline
\end{tabular}

Table 3 Results of study on amino acid profiles in elderly patients divided into two groups: non-frail and severely frail (Adachi et al. 2018)

\begin{tabular}{llll}
\hline Authors & Year & Results & Comments \\
\hline Adachi et al. 2018 & $\begin{array}{l}\text { Significant increase in concentration of glutamine in patients with SF comparing to NF } \\
\text { group }\end{array}$ & $\begin{array}{l}\text { Non-frail (NF) group-N=28 } \\
\text { Severely frail (SF) group- }-N=31\end{array}$ \\
& $\begin{array}{l}\text { Significant decrease in concentration of tyrosine, arginine, phenylalanine, histidine, } \\
\text { isoleucine, leucine, lysine, methionine, threonine, tryptophan, valine in patients with }\end{array}$ & \\
& SF comparing to NF group &
\end{tabular}




\section{Amino acid profiling in patients with different types of neurodegenerative diseases}

Amino acids play essential roles in the regulation of key functions of the human body. Physiological amino acid concentration depends on the organs' functions and pathological conditions may change their metabolism. Recently, researchers have shown an increased interest in amino acids profiling, especially their potential application in early diagnosis of numerous diseases. A number of studies have found differences between the level of amino acid profiling in patients with various types of diseases and in healthy control group, for example in patients with Alzheimer's disease (Assche et al. 2015; Bateman et al. 2012; Corso et al. 2017; Gong et al. 2015; Inoue et al. 2013; Fonteh et al. 2007), Parkinson's disease (Blesa et al. 2015; Figura et al. 2018) and elderly patients (Adachi et al. 2018). Recent evidence suggests that metabolic factors can play key roles in the neurodegenerative process, at least in the early stage in the pathogenic process (Ngadu et al. 2015; Bredesen 2015). Neurochemistry is defining the levels of amino acids pathways in brain. For example, glutamate, glycine and bridge peptides have neuroactive properties and they are neurotransmitters. Glutamine is in the central metabolic pathway. Its most important role in the brain is that of a precursor of the neurotransmitter amino acids: the excitatory amino acids, glutamate and aspartate, and the inhibitory amino acid, $\gamma$-amino butyric acid. Based on some works, the metabolism of neurotransmitters (NTs) and their metabolites in body fluids are significantly different between healthy people and patients with neurodegenerative diseases such as AD. The group of NTs includes amino acid neurotransmitters and monoamine neurotransmitters. Both of them are widely distributed and could play important roles in the activities of peripheral and nervous systems. Accordingly, the comprehensive analysis and qualification of neurotransmitters and their metabolites would provide a significant clinical value in development of neurodegenerative diseases diagnosis. The alterations in the effects of neurotransmitters may be responsible for numerous pathologies or may be a function of normal aging (Zhao et al. 2017; Gong et al. 2015; Lv et al. 2015).

\section{PFAA profiles among patients with Alzheimer's disease (AD)}

Based on the World Alzheimer Report (2009) the AD is the most common cause of dementia in the world (Assche et al. 2015). It is an incurable, progressive and fatal disorder that causes problems with memory, thinking, perception and behavior among older patients. Most patients are diagnosed at a late stage of the disease, because the symptoms of AD can be easily mistaken with aging. In previous studies, researchers demonstrated that disease's process begins more than 30 years prior to clinical symptoms of dementia and is related to series of changes that occur over many years in cerebrospinal fluid biomarkers appropriate to plaques of amyloid beta, tangles of tau protein, brain metabolism and progressive cognitive disorders (Inoue et al. 2013; Bateman et al. 2012; Mattsson et al. 2012). It is necessary to identify the association between metabolic processes and disease's pathology.

Nai-jing et al. investigated plasma metabolites concentrations in 20 patients with $\mathrm{AD}$ and 20 healthy control subjects. Free-of-dementia volunteers in healthy group did not have any neurological and cognitive disease. Researchers have indicated that concentration of tryptophan (Trp) has significantly decreased in patients with AD compared to healthy controls (Nai-jing et al. 2010).

Further study conducted by Corso et al. has provided more evidence of AD-related changes in serum amino acid profile. They investigated serum in four groups of patients-29 participants with potential AD, 18 patients with mild cognitive impairment (MCI), 24 patients with subjective memory complaint (SMC) and 46 healthy subjects. They found a progressive decrease in glutamate, aspartate, and phenylalanine concentration and a progressive in concentrations of citrulline, argininosuccinate and homocitrulline, from healthy group over SMC and MCI to AD. Researchers have shown the impact of disorder progression for the amino acids profile. The study indicated potential of using amino acids profiling for the monitoring in elderly patients to detect at-risk subjects. This demonstrated the possible use of plasma-free amino acid profiling in early detection of Alzheimer's disease (Corso et al. 2017).

Fonteh et al. enrolled eight AD patients and eight healthy controls to their study. There was no significant age difference in both groups. They reported a significantly lower concentration of histidine in cerebrospinal fluid from $\mathrm{AD}$ patients compared to the control group. Furthermore, researchers reported that in urine, the level of histidine, 3-methyl-histidine and carnosine (dipeptide molecule, made up of the amino acids betaalanine and histidine) was significantly higher in patients with AD than in control group. The concentrations of 1-methylhistidine and anserine were significantly lower in patients with $\mathrm{AD}$ urine. Concentrations of total histidine and methyl-histidine were significantly higher in patients with $A D$ urine and level of total carnosine and anserine was lower in urine from patients with $\mathrm{AD}$. The results of this investigation show that precursors of carnosine and anserine are lower in cerebrospinal fluid and plasma. Authors of this paper indicated that further studies are needed to define and validate the causes of these changes in cerebrospinal fluid and plasma. In presented study, 
the concentration of aromatic-containing amino acids was also analyzed. They detected that level of plasma L-DOPA was significantly higher in patients with AD material than in healthy subjects. In contrast, the concentration of plasma dopamine was lower in patients with $\mathrm{AD}$ material than in healthy control group. Furthermore, the ratio of L-DOPA to tyrosine was significantly higher in material from patients with $\mathrm{AD}$ compared to control group while the ratio of dopamine to L-DOPA was significantly lower in material from patients with AD than in control group. Differences in enzyme substrates and their products ratios are shown. These results suggest the changes in tyrosine hydrolase and L-DOPA decarboxylase activities in patients' with $\mathrm{AD}$ material. Summarizing, these results present changes in peripheral dopamine biosynthesis in material from patients with $\mathrm{AD}$ which are probably assigned to enzymes in the dopamine synthetic pathway (Fonteh et al. 2007).

\section{AA profiles among patients with Parkinson's disease}

Parkinson disease (PD) is one of the most common chronic, progressive neurological diseases. This disorder is associated with a loss of dopaminergic neurons in the substantia nigra pars compacta in the humans brain. It is a disease of still unknown origin (Blesa et al. 2015). Prevalence of Parkinson's disease increases with age. More than $1 \%$ of population above 60 years is affected by PD. PD appears as a movement disorder with three main symptoms: tremor, rigidity and bradykinesia (Tysnes and Storstein 2017).

Figura et al. conducted a study on 73 patients suffering on Parkinson's disease (22 patients in early PD group, 51 patients in advanced PD group). They observed significant differences in the level of alanine, arginine, threonine and phenylalanine. All listed amino acids were significantly lower in patients with advanced Parkinson's disease than in patients on early stage of disease. Researchers reported no significant differences regarding other analyzed amino acids. According to the researchers' conclusions, the possible mechanisms of serum amino acids concentration changes in Parkinson's disease include results of oxidative stress, effects of mitochondrial dysfunction, malabsorption and changes in amino acid metabolism, effects of progressive neurodegenerative processes in the brain and results of aromatic L-amino decarboxylase inhibitors and dopaminergic medications (Figura et al. 2018).

\section{AA profiles in elderly patients}

Significant changes in the demographic structure of most European countries are resulting in more and more consequences. Aging is associated with numerous physiologic changes and an increased susceptibility to diseases. Age is the greatest single risk factor for numerous neurological diseases. Researchers try to find answers to many questions about human organism to properly protect the aging society. The most important challenges are comprehensive understanding of the causal molecular and environmental mechanisms, molecular processes that influence aging and longevity, environmental processes that influence longevity and age-related diseases and finally efforts to identify predictive factors or biomarkers of age-related diseases. In recent years, researchers have shown an increasing interest in biomarkers of aging. These factors should help to define and characterize the biological age and, as age is one of the main risk factors in many diseases, could be then used to identify patients at high risk of age-related diseases and disability occurrence (Simm et al. 2008; Hoffman et al. 2017).

Adachi et al. have analyzed plasma amino acid profile on 59 elderly patients in Japan divided into two groups-nonfrail $(n=28)$ and severely frail $(n=31)$. Frailty was assessed using the CSHA-CFS Scale, a global clinical measure of biological age. This scale includes seven points with predictive validity for clinical judgment (mortality and prognostic power). Researchers observed significant differences between the non-frail (NF) and severely frail (SF) groups for 11 plasma-free amino acids-glutamine, tyrosine and all essential amino acids, EAA). Glutamine was higher and tyrosine was lower in the SF group compared to NF group. All plasma EAAs were significantly lower in the SF patients group compared to NF group. Furthermore, they indicated strong correlations between plasma amino acids (leucine, valine, lysine tryptophan, total EAA levels) and BMI, albumin and prealbumin levels (Adachi et al. 2018).

\section{Discussion}

During recent years the results of numerous scientific papers indicate the potential of using amino acids profiling for improving diagnosis of various diseases, including neurodegenerative diseases (Nai-jing et al. 2010; Corso et al. 2017; Fonteh et al. 2007; Figura et al. 2018; Adachi et al. 2018). In this paper, research of plasma amino acid profiling was reviewed to assess whether amino acids can be used for diagnosis of various types of neurodegenerative diseases. Presented studies showed that the levels of several plasma amino acids have changed significantly between healthy subjects and patients with neurodegenerative diseases. This paper indicates the potential application of the amino acid detection methods as a diagnostic tool for $\mathrm{AD}, \mathrm{PD}$, and for determination of biomarkers of aging. Further analysis is required to determine the specificity and sensitivity of indicated biomarker candidates. 


\section{Compliance with ethical standards}

Conflict of interest The authors declare that they have no conflict of interest.

Research involving human participants and/or animals For this type of study formal consent is not required. This article does not contain any studies with human participants or animals performed by any of the authors.

Informed consent Informed consent obtaining for this type of study is not required.

OpenAccess This article is distributed under the terms of the Creative Commons Attribution 4.0 International License (http://creativeco mmons.org/licenses/by/4.0/), which permits unrestricted use, distribution, and reproduction in any medium, provided you give appropriate credit to the original author(s) and the source, provide a link to the Creative Commons license, and indicate if changes were made.

\section{References}

Adachi Y, Ono N, Imaizumi A, Muramatsu T, Andou T, Shimodaira Y, Nagao K, Kageyama Y, Mori M, Noguchi Y, Hashizume N, Nukada H (2018) Plasma amino acid profile in severely frail elderly patients in Japan. Int J Gerontol. https://doi.org/10.1016/j. ijge.2018.03.003

Assche R, Temmerman L, Dias DA, Boughton B, Boonen K, Braeckman BP, Schoofs L, Roessner U (2015) Metabolic profiling of a transgenic Caenorhabditis elegans Alzheimer model. Metabolomics 11(2):477-486

Bateman RJ, Xiong C, Benzinger TL, Fagan AM, Goate A, Fox NC, Marcus DS, Cairns NJ, Xie X, Blazey TM, Holtzman DM, Santacruz A, Buckles V, Oliver A, Moulder K, Aisen PS, Ghetti B, Klunk WE, McDade E, Martins RN, Masters CL, Mayeux R, Ringman JM, Rossor MN, Schofield PR, Sperling RA, Salloway S, Morris JC (2012) Clinical and biomarker changes in dominantly inherited Alzheimer's disease. N Engl J Med 367(9):795-804

Blesa J, Trigo-Damas I, Quiroga-Varela A, Jackson-Lewis VR (2015) Oxidative stress and Parkinson's disease. Front Neuroanat 9(91):1-9

Bredesen DE (2015) Metabolic profiling distinguishes three subtypes of Alzheimer's. Aging 7(8):595-600

Corso G, Cristofano A, Sapere N, Marca G, Angiolillo A, Vitale M, Fratangelo R, Lombardi T, Porcile C, Intrieri M, Costanzo A (2017) Serum amino acid profiles in normal subjects and in patients with or at risk of Alzheimer dementia. Dement Geriatr Cogn Disord Extra 7:143-159

Figura M, Kuśmierska K, Bucior E, Szlufik S, Koziorowski D, Jamrozik Z, Janik P (2018) Serum amino acid profile in patients with Parkinson's disease. PLoS ONE 13(1):1-12

Fonteh AN, Harrington RJ, Tsai A, Liao P, Harrington MG (2007) Free amino acid and dipeptide changes in the body fluids from Alzheimer's disease subjects. Amino Acids 32:213-224

Gong Y, Liu Y, Zhou L, Di X, Li W, Li Q, Bi K (2015) A UHPLCTOF/MS method based metabonomic study of total ginsenosides effects on Alzheimer disease mouse model. J Pharm Biomed Anal 10(115):174-182
Hasim A, Aili A, Maimaiti A, Mamtimin B, Abudula A, Upur H (2013) Plasma-free amino acid profiling of cervical cancer and cervical intraepithelial neoplasia patients and its application for early detection. Mol Biol Rep 40(10):5853-5859. https://doi. org/10.1007/s11033-013-2691-3

Hoffman JM, Lyu Y, Pletcher SD, Promislow DE (2017) Proteomics and metabolomics in ageing research: from biomarkers to systems biology. Essays Biochem 61(3):379-388

Hui-Ming G, Jau-Shyong H (2008) Why neurodegenerative diseases are progressive: uncontrolled inflammation drives disease progression. Trends Immunol 29(8):357-365

Inoue K, Tsutsui H, Akatsu H, Hashizume Y, Matsukawa N, Yamamoto T, Toyo'oka T (2013) Metabolic profiling of Alzheimer's disease brains. Sci Rep 3:2364. https://doi.org/10.1038/srep02364

Lv C, Li Q, Liu X, He B, Sui Z, Xu H, Yin Y, Liu R, Bi K (2015) Determination of catecholamines and their metabolites in rat urine by ultra-performance liquid chromatography-tandem mass spectrometry for the study of identifying potential markers for Alzheimer's disease. J Mass Spectrom 50(2):354-363

Mattsson N, Rosén E, Hansson O, Andreasen N, Parnetti L, Jonsson M, Herukka SK, van der Flier WM, Blankenstein MA, Ewers M, Rich K, Kaiser E, Verbeek MM, Olde Rikkert M, Tsolaki M, Mulugeta E, Aarsland D, Visser PJ, Schröder J, Marcusson J, de Leon M, Hampel H, Scheltens P, Wallin A, Eriksdotter-Jönhagen M, Minthon L, Winblad B, Blennow K, Zetterberg H (2012) Age and diagnostic performance of Alzheimer disease CSF biomarkers. Neurology 78(7):468-476

Nai-jing L, Wen-tao L, Wei L, Sheng-qi L, Xiao-hui C, Kai-shun B, Ping H (2010) Plasma metabolic profiling of Alzheimer's disease by liquid chromatography/mass spectrometry. Clin Biochem 43:992-997

Ngadu T, Lehtisalo J, Solomon A, Levalahti E, Ahtiluoto S, Antikainen R, Backman L, Hanninen T, Jula A, Laatikainen T, Lindstrom J, Mangialasche F, Paajanen T et al (2015) A 2 year multidomain intervention of diet, exercise, cognitive training, and vascular risk monitoring versus control to prevent cognitive decline in at-risk elderly people (FINGER): a randomized controlled trial. Lancet 385:2255-2263

Roth C, Kasimir-Bauer S, Pantel K, Schwarzenbach H (2011) Screening for circulating nucleic acids and caspase activity in the peripheral blood as potential diagnostic tools in lung cancer. Mol Oncol 5(3):281-291. https://doi.org/10.1016/j.molonc.2011.02.002

Simm A, Nass N, Bartling N, Hofmann B, Silber RE, Santos AN (2008) Potential biomarkers of ageing. Biol Chem 389:257-265

Tysnes OB, Storstein A (2017) Epidemiology of Parkinson's disease. J Neural Transm (Vienna) 124(8):901-905

World Alzheimer Report (2009) Alzheimer's disease international. https://www.alz.co.uk/research/files/WorldAlzheimerReport.pdf. Accessed 21 Sept 2018

Zhao XE, He Y, Li M, Chen G, Wei N, Wang X, Sun J, Zhu S, You J (2017) Analysis of amino acid and monoamine neurotransmitters and their metabolites in rat urine of Alzheimer's disease using in situ ultrasound-assisted derivatization dispersive liquid-liquid microextraction with UHPLC-MS/MS. J Pharm Biomed Anal 20(135):186-198

Publisher's Note Springer Nature remains neutral with regard to jurisdictional claims in published maps and institutional affiliations. 